Prepared for the U.S. Department of Energy

Under Contract DE-AC05-76RL01830

\title{
Preliminary Report on the Evaluation of an Electron- Positron Collider as a source of Monoenergetic Photons
}

JE Fast, LW Campbell (PNNL)

November 30, 2009

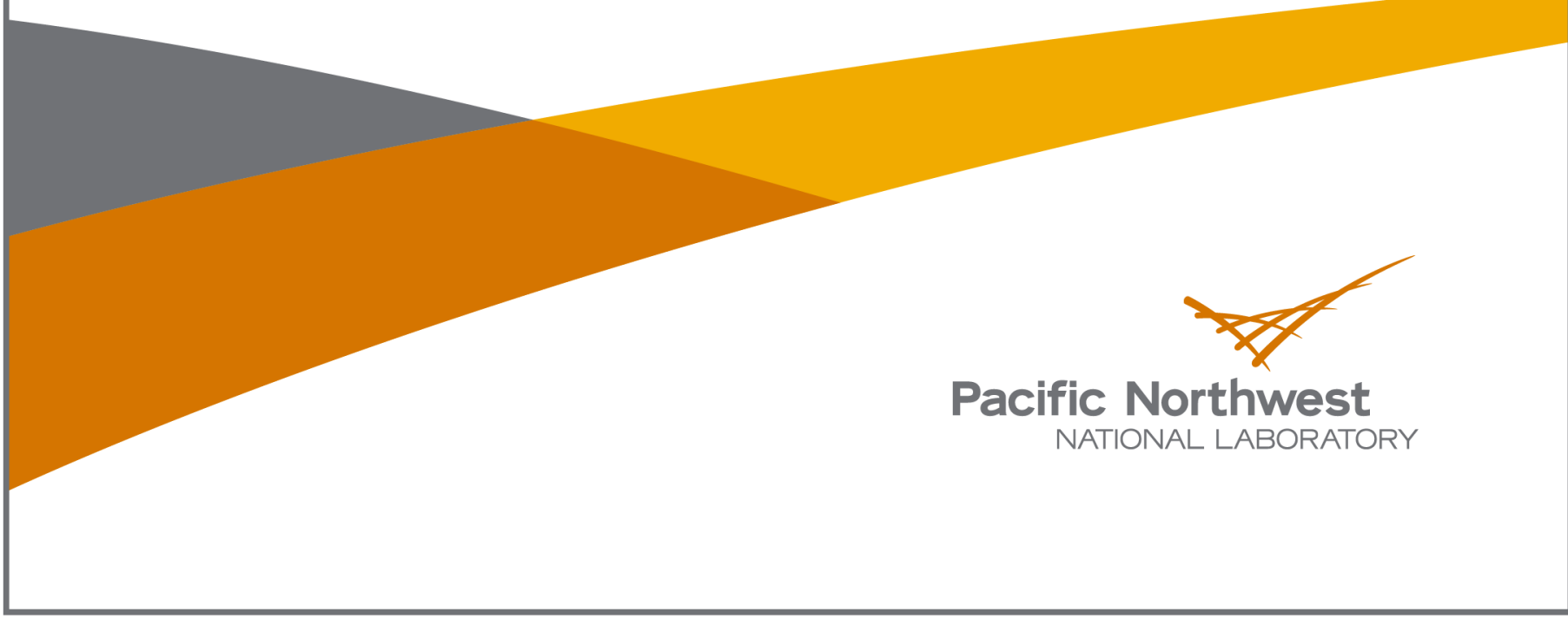




\title{
DISCLAIMER
}

This report was prepared as an account of work sponsored by an agency of the United States Government. Neither the United States Government nor any agency thereof, nor Battelle Memorial Institute, nor any of their employees, makes any warranty, express or implied, or assumes any legal liability or responsibility for the accuracy, completeness, or usefulness of any information, apparatus, product, or process disclosed, or represents that its use would not infringe privately owned rights. Reference herein to any specific commercial product, process, or service by trade name, trademark, manufacturer, or otherwise does not necessarily constitute or imply its endorsement, recommendation, or favoring by the United States Government or any agency thereof, or Battelle Memorial Institute. The views and opinions of authors expressed herein do not necessarily state or reflect those of the United States Government or any agency thereof.

\author{
PACIFIC NORTHWEST NATIONAL LABORATORY \\ operated by \\ BATTELLE \\ for the \\ UNITED STATES DEPARTMENT OF ENERGY \\ under Contract DE-AC05-76RL01830
}




\section{Preliminary Report on the Evaluation of an Electron-Positron Collider as a source of Monoenergetic Photons}

JE Fast, LW Campbell (PNNL)

November 30, 2009

Prepared for

the U.S. Department of Energy

under Contract DE-AC05-76RL01830

Pacific Northwest National Laboratory

Richland, Washington 99352 

PNNL- SA-70285

\section{Abstract}

Active interrogation methods are being investigated to detect shielded special nuclear material (SNM). These approaches utilize either neutron or photon beams to excite the SNM in concert with either neutron or gamma ray detectors to observe the stimulated emissions. The two primary methodologies with photon beams are photofission and nuclear resonance florescence (NRF). Photofission requires photons energies of 7-10 MeV while NRF requires photon energies around $2 \mathrm{MeV}$. For both techniques, photons that are not in the appropriate energy band, e.g. the low energy tail of a Bremsstrahlung photon beam, contribute unwanted additional radiation dose to cargo. Typically less than $10 \%$ of the photons are in the usable energy band. The additional photon production generates a commensurate amount of additional radiation dose in the source and target areas, impacting shielding requirements and/or dose to operators and equipment and at the expense of a similar increase in power consumption. Hence it is highly desirable to produce narrow energy ("monoenergetic") photon beams with tunable energy in the range of $\sim 2-20 \mathrm{MeV}$.

This study investigates a novel approach to producing monoenergetic, tunable photon beams in which electrons and positrons in collinear, equal energy beams at zero relative velocity annihilate to produce gamma rays. In the center-of-mass frame the annihilation produces a pair of $511 \mathrm{keV}$ photons back-to-back. These are Lorentz boosted in the lab frame producing a photon beam peaked in the forward direction (i.e. the direction of flight of the e+ and e- beams) with a peak energy equal to twice the beam energy (i.e. the sum of the e+ and eenergies). The photon energy spread in the core of the beam is a function of the Lorentz boost related to beam energy and becomes rather small at energies of a few MeV. Furthermore, there is a complete correlation of photon energy and angle so collimation can be used to produce a narrower energy spread beam. In contrast, there is no such correlation in a Bremsstrahlung beam, and hence no practical way to suppress the undesirable low energy tail of the beam. Alternative and more conventional configurations of head-on collisions and positron beams incident on a low- $Z$ fixed target will also be investigated.

In this report we first discuss the basic physics of the approach and conceptual design of such a photon source. We then discuss the research issues that must be addressed to assess the feasibility of a photon source based on this technical approach. We conclude with an outline of the research path going forward from this point. I critical element of the research involves a subcontract to Muons, Inc., a small business that brings decades of accelerator design experience in high brightness beams, anti-matter beam production, and industrial accelerators to the project. 

PNNL- SA-70285

\section{Executive Summary}

Detection of illicit transport of special nuclear materials (SNM) in quantities of interest, e.g. IAEA quantities of concern, is extremely challenging using passive methods. Of particular concern is highly enriched uranium, which has radiation emissions that are easily shielded. To meet this challenge, methods of active interrogation by high energy photons are being investigated. High energy photons can penetrate deeply and will cause SNM to produce highly penetrating radiations that can overcome shielding. Two types of active photon scanning are considered promising: Nuclear resonance fluorescence in which incident photons of a specific energy are absorbed and re-emitted from the SNM; and photofission in which the photon causes the SNM to undergo fission, releasing neutrons and gamma rays. Photofission requires higher energy photons than nuclear resonance fluorescence, but is less selective in terms of the necessary photon energy.

Photons that are not at useful energies for the scanning technique being employed do not contribute to the signal, but do add unwanted radiation dose to the target being scanned. In addition, radiation scattered or emitted from the target during the scanning process irradiates the surrounding area, increasing the shielding requirements for the operators. An ideal photon source would thus only produce photons with the necessary energy for the scanning technique to maximize the signal, reduce interference, reduce power consumption, and reduce the total radiation dose. Current photon sources do not meet this criterion, producing instead photons over a wide energy range most of which are not useful as a result of being outside the energy band for scanning.

In this report, we propose a particle accelerator that brings together electrons with their antimatter counterparts, positrons. When electrons meet positrons, they annihilate each other and produce a pair of gamma ray photons at a specific energy. Unlike the more familiar electron/positron colliders used in particle physics research, in this configuration the beams are not colliding head on. Instead, they are brought together moving in the same direction so that the beams collide in a collinear collision at low or zero relative velocity. The energy of the photons emitted can be changed by varying the energy of the electron and proton beams. This allows the photon source to be adapted to the scanning requirements while minimizing the radiation produced. When tuned to photofission energies, essentially all the photons will be potentially capable of inducing photofission. For nuclear resonance fluorescence, the beam energy spread will exceed the narrow width of the nuclear resonance being probed, but by restricting the emitted photon energies to a narrow band around the resonance energy the radiation dose can be reduced by several orders of magnitude and improving the signal to noise ratio by a smaller but still large factor.

There do not appear to be any fundamental reasons that annihilation cannot produce a clean, tunable beam of nearly mono-energetic photons. There are, however, a number of technical issues that must be explored to determine if an annihilation photon source is feasible compared to other possible techniques to produce a monoenergetic gamma ray beam. There are a number of possible methods of producing and collecting the positrons, several options for allowing the positrons to interact with electrons so as to select photons of the desired energy, 
and engineering issues such as the best way to accelerate and focus the beams for annihilation. If enough positrons can be created and gathered, annihilation can be a feasible photon source for active interrogation. 


\section{Contents}

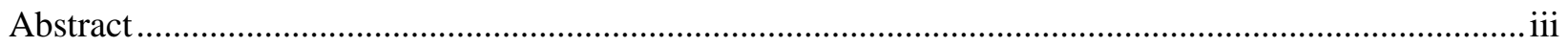

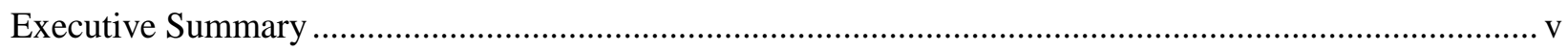

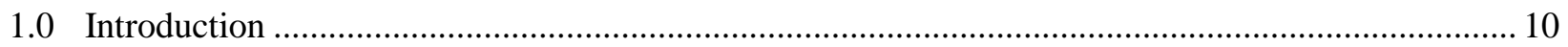

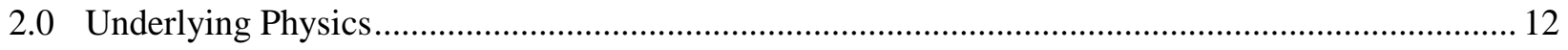

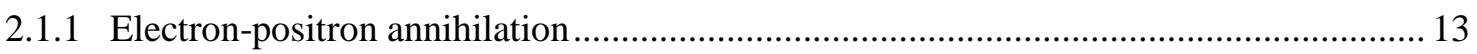

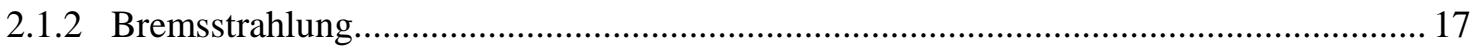

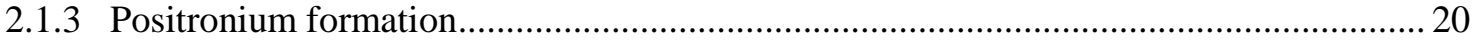

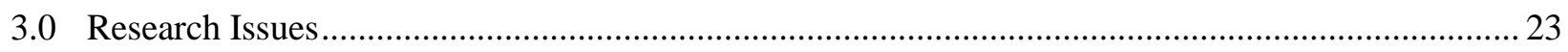

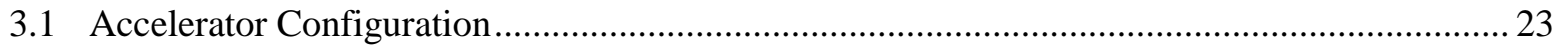

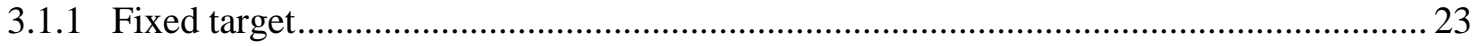

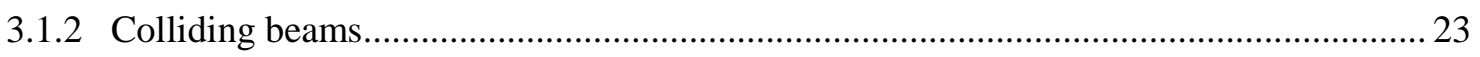

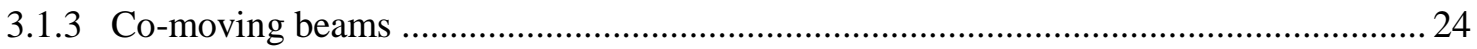

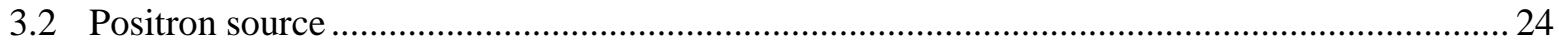

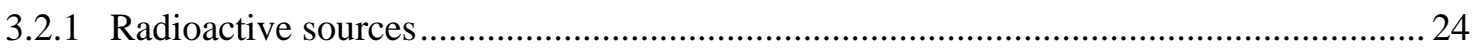

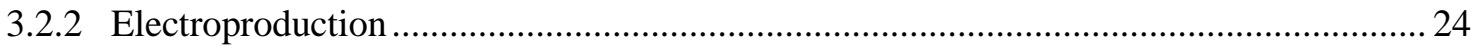

3.2.3 Proton beam produced short-lived positron sources .................................................. 25

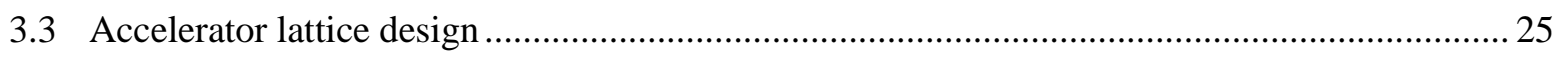

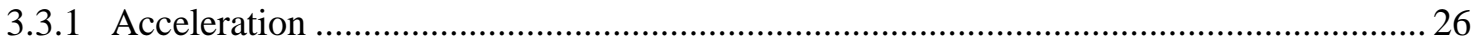

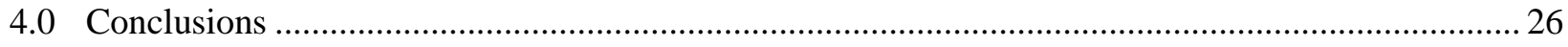

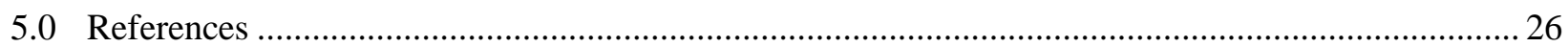




\section{Figures}

Figure 1: Photon spectrum for $10 \mathrm{MeV}$ positrons striking a fixed beryllium target. In this configuration both Bremsstrahlung and annihilation occur. With collinear beams the Bremsstrahlung, or "slowing down" radiation, is suppressed leaving just the annihilation peak.

Figure 2: Schematic layout of the proposed photon source. ......................................................... 12 

PNNL- SA-70285

\subsection{Introduction}

Detection of illicit transport of special nuclear materials (SNM) in quantities of interest, e.g. IAEA quantities of concern, is extremely challenging using passive methods. Of particular concern is HEU which has easily shielded gamma ray emissions and no significant neutron emission rate. To meet this challenge, active interrogation methods are being investigated. These approaches can utilize either neutron or photon beams to stimulate the SNM in concert with either neutron or gamma ray detectors to observe the stimulated emissions. The two primary methodologies that employ photon beams are photofission and nuclear resonance florescence (NRF). The former requires photons in the range of $10 \mathrm{MeV}$ while the latter would use photons near $2 \mathrm{MeV}$. For both techniques, photons that are not in the appropriate energy band, e.g. the low energy tail of a Bremsstrahlung photon beam, contribute unwanted additional radiation dose to cargo. In addition, the total photon production rate is typically an order of magnitude (or more) greater than the rate required at the energy of interest. This additional photon production generates a commensurate amount of additional radiation dose in the source and target areas, impacting shielding requirements and/or dose to operators and equipment and at the expense of a similar increase in power consumption. Hence it is highly desirable to produce narrow energy ("monoenergetic") photon beams with tunable energy in the range of $\sim 2$ $20 \mathrm{MeV}$.

This study investigates a novel approach to producing monoenergetic, tunable photon beams using the annihilation of electrons and positrons in collinear, equal energy beams colliding at near-zero relative velocity. In the center-of-mass frame the annihilation produces a pair of $511 \mathrm{keV}$ photons back-to-back. These photons are Lorentz boosted in the lab frame and the energy of these photons is determined by the kinematics of the pair in the center of momentum frame and the transformation of the photon momentum to the laboratory frame. For example, when the momentum of the pair is aligned with the beam axis, the forward going photon is boosted to a peak gamma ray energy approximately equal to twice the beam energy (i.e. the sum of the e+ and e- energies). The photon energy spread in the core of the beam is a function of the Lorentz boost (i.e. beam energy) and becomes rather small at energies of a few $\mathrm{MeV}$. Furthermore, there is a complete correlation of photon energy and direction so collimation can be used to select a narrower energy spread beam. In contrast, there is no such correlation in a Bremsstrahlung beam, and hence no clean method to suppress the undesirable low energy tail of the beam.

The principle of positron annihilation in flight to produce a narrow energy spread beam of photons has been around since the 1960's, however earlier efforts have used a positron beam incident on a foil target [Caldwell et al, 1980]. The resulting photon energy spectrum, shown in Figure 1, contains both the annihilation component near the end-point of the energy spectrum and the much larger Bremsstrahlung contribution from the interactions of the positrons in the foil target. The Bremsstrahlung (this translates roughly to "slowing down") contribution originates from radiative interactions of the fast moving positrons with the electromagnetic field of the nuclei in the target foil. This is the same mechanism currently used to produce nearly all photon beams for active interrogation. A similar interaction (beamstrrahlung) occurs in colliding beam accelerators where the electromagnetic field is generated by the dense electron beam cloud rather than nuclei. The approach pursued in this research aims to eliminate the Bremsstrahlung 
contribution by reducing the relative velocity of the positrons and electrons (and hence the electromagnetic field created by the electrons) to near zero. In the case of equal energy, collinear beams the remaining relative velocity is due to transverse and longitudinal energy spread of the beams, typically controllable to of order $10 \mathrm{keV}$ for beams in the energy range of interest.

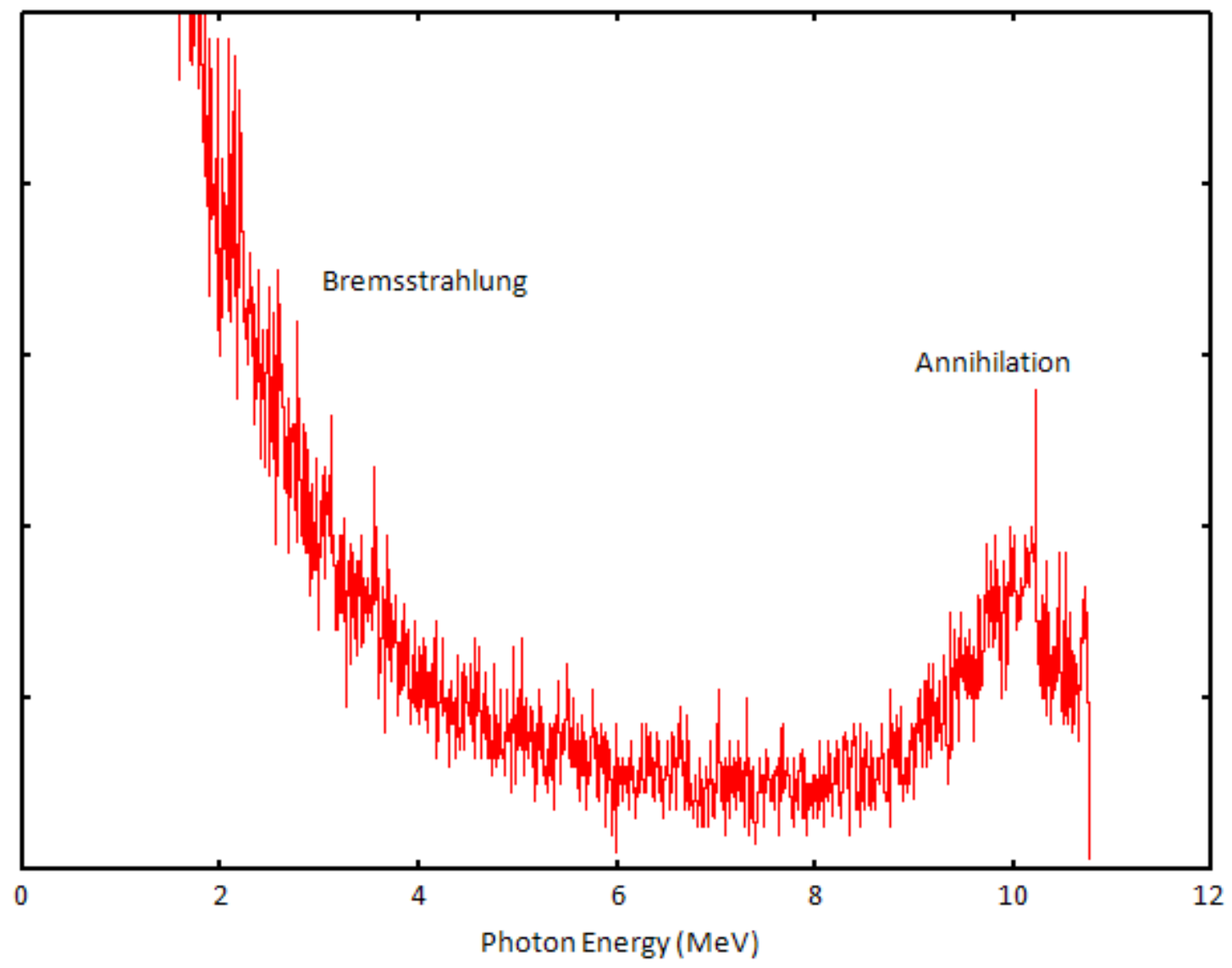

Figure 1: Photon spectrum for $10 \mathrm{MeV}$ positrons striking a fixed beryllium target. In this configuration both Bremsstrahlung and annihilation occur. With collinear beams the Bremsstrahlung, or "slowing down" radiation, is suppressed leaving just the annihilation peak.

The concept of a collinear, equal energy e+e- collider has been proposed for studies of positronium and Fermi degeneracy in ultra-cold electron beams [Mikhailichenko 2003, Mikhailichenko 1996]. These studies require extremely low emittance (a measure of beam transverse energy spread), ultra-cold (i.e. very low energy spread) beams. For example, the binding energy of positronium is only a few $\mathrm{eV}$, much higher than the typical energy spread of a few MeV electron or positron beam. The LEPTA facility [E. Ahmanova et al 2009] for positronium research utilizing this technique has recently been commissioned at the Dubna research laboratory in Russia. This facility operates at an energy of only $10 \mathrm{keV}$ in order to reduce the beam energy spread to a point where the positronium bound state formation becomes the favored interaction process. 
A schematic layout of the accelerator configuration to produce the monoenergetic, tunable photon beam is shown in Figure 2. The total photon flux will be determined by the number of electrons, the number of positrons, the beam emittances and the relative velocity of the particles while they traverse the interaction region. The most obvious limitation on the photon flux will be the positron production rate. Therefore it will be essential to efficiently store the positrons in a recirculating accelerator, as shown. Electrons are much easier to produce and hence it is possible that a single pass configuration could be used for the electron beam, as shown in the sketch.

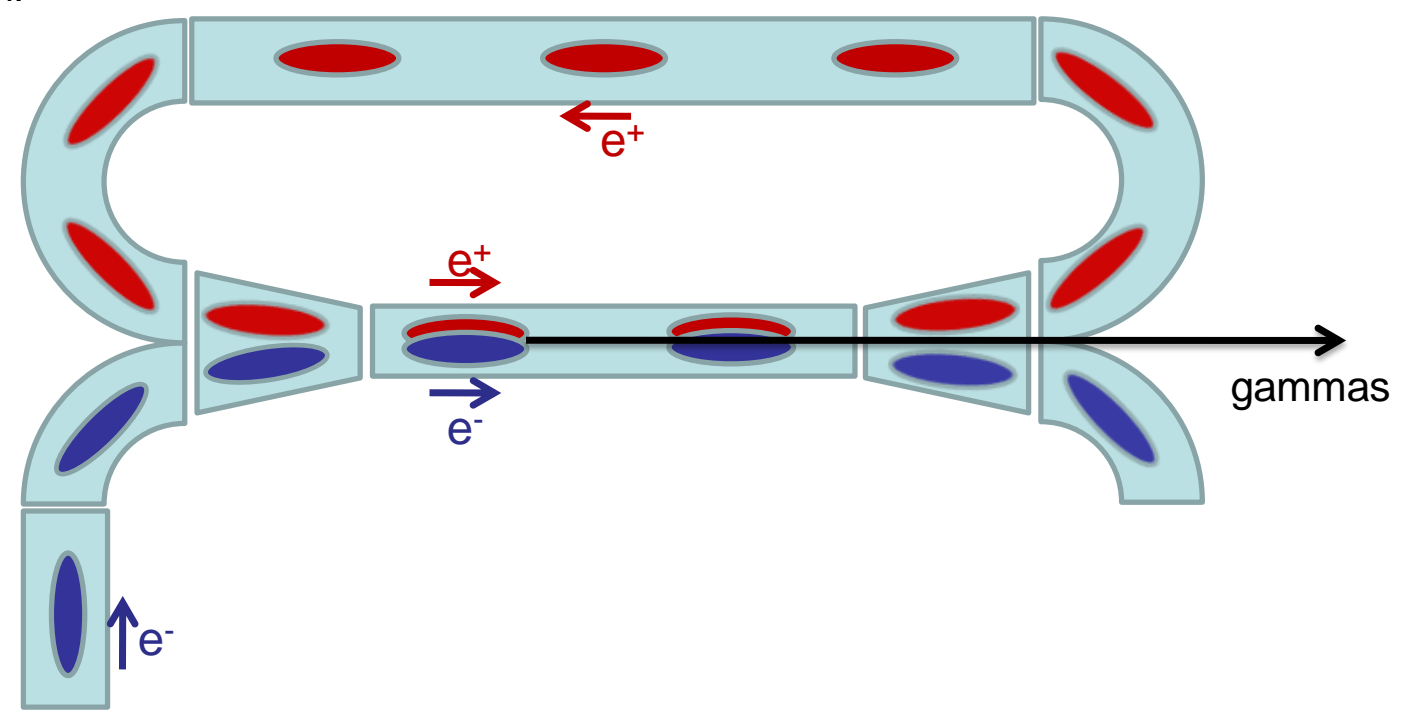

Figure 2: Schematic layout of the proposed photon source.

\subsection{Underlying Physics}

This section describes the underlying physics upon which the technique is based. Electrons and positrons will interact in several ways when the beams collide. The rate of interaction is expressed as the product $\mathcal{L} \sigma$, where $\mathcal{L}$ is the accelerator luminosity and $\sigma$ is the relevant cross section, typically expressed in units of barns $\left(10^{-24} \mathrm{~cm}^{2}\right)$. There are three relevant processes for the accelerator configuration proposed here.

1. Electron-positron annihilation

2. Bremsstrahlung

3. Positronium formation 
PNNL- SA-70285

\subsubsection{Electron-positron annihilation}

Consider a spin-unpolarized electron and positron approaching each other in their center-ofmass frame, with velocity $v$ and Lorentz factors $\beta=v / c$ and $\gamma=1 / \sqrt{1-(v / c)^{2}}$ where $c$ is the speed of light in vacuum. The differential cross section for electron-positron annihilation into two photons at angle $\theta$ from the original axis of motion is

$$
\begin{aligned}
\frac{d \sigma_{a}}{d \Omega}=\left(\frac{r_{e}}{4 \gamma}\right)^{2} \frac{1}{\beta}\{ & -\left[\frac{1}{\gamma^{2}(1-\beta \cos (\theta))}+\frac{1}{\gamma^{2}(1+\beta \cos (\theta))}\right]^{2} \\
& \left.+2\left[\frac{1}{\gamma^{2}(1-\beta \cos (\theta))}+\frac{1}{\gamma^{2}(1+\beta \cos (\theta))}\right]+\left[\frac{1+\beta \cos (\theta)}{1-\beta \cos (\theta)}+\frac{1-\beta \cos (\theta)}{1+\beta \cos (\theta)}\right]\right\}
\end{aligned}
$$

where $r_{e}$ is the classical electron radius.

At low energies, the photon distribution is nearly independent of angle. At highly relativistic energies, $\beta$ is approximately 1 and the cross section for annihilation is strongly peaked in the forward and reverse direction. 
PNNL- SA-70285

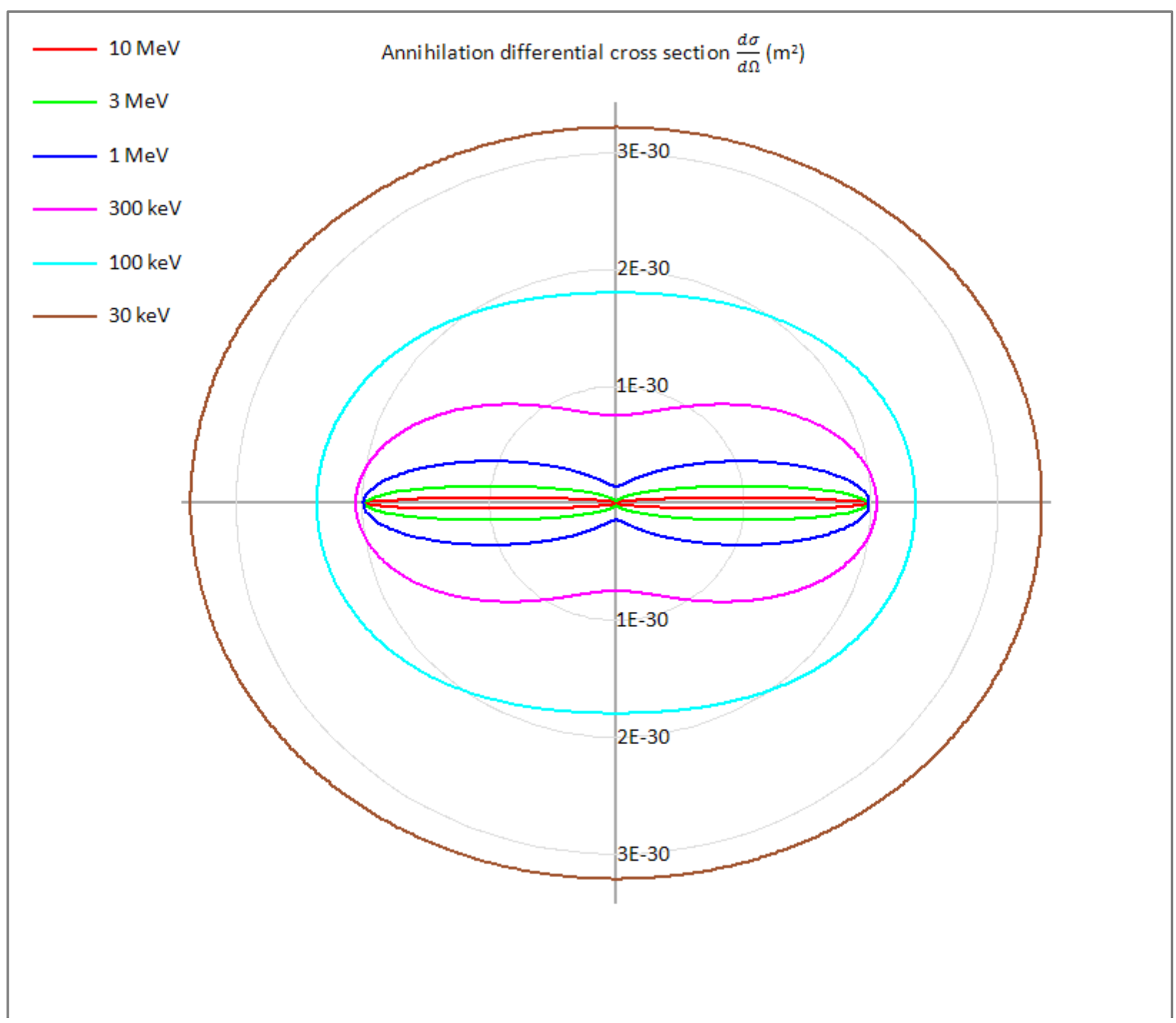

Figure 3: Angular differential cross section for annihilation, shown as a polar plot with respect to angle. The beam is directed along the $x$-axis.

In the rest frame of the electron, a positron approaching at relative velocity $v_{\text {rel }}$ and Lorentz $\gamma=1 / \sqrt{1-\left(v_{\text {rel }} / c\right)^{2}}$ has a total two-photon annihilation cross section of:

$$
\sigma_{a}=\frac{\pi r_{e}^{2}}{\gamma+1}\left[\frac{\gamma^{2}+4 \gamma+1}{\gamma^{2}-1} \ln \left(\gamma+\sqrt{\gamma^{2}-1}\right)-\frac{\gamma+3}{\sqrt{\gamma^{2}-1}}\right]
$$

[Dirac 1928]. In the limit of low relative velocity this reduces to $\pi r_{e}^{2} c / v_{\text {rel }}$. All other annihilation channels are over two orders of magnitude less probable [Ore and Powell, 1949].

A positron traversing an electron gas will have an inverse annihilation lifetime (probability of annihilation per unit time) of $\tau^{-1}=v_{\text {rel }} \rho_{e} \sigma_{a}$, where $\rho_{e}$ is the electron density. This reduces to $\tau^{-1}=\pi \rho_{e} r_{e}^{2} c$, which is independent of velocity. Therefore, for a bunch of positrons with density $\rho_{p}$ co-propagating with a bunch of electrons at temperatures $T \ll m_{e} c^{2} / k_{B}$ (where $m_{e}$ 
is the electron rest mass and $k_{B}$ is the Boltzmann constant), the rate of photon production per unit volume in the rest frame of the bunch is $d^{2} n_{p h} /(d t d V)=2 \pi \rho_{p} \rho_{e} r_{e}^{2} c$, where the factor of two arises because each annihilation produces two photons.

In the lab frame of reference, this rate is reduced by a factor $1 / \gamma^{2}$ - the number of photons is a relativistic invariant and the differential four-volume $d t d V$ is unchanged by a boost, while the number densities computed in the lab frame will be a factor of $\gamma$ larger than those in the bunch rest frame due to relativistic length contraction. We can thus estimate the rate at which annihilation photons are produced by a co-propagating bunch of $N_{p}$ positrons in $N_{e}$ electrons of total (lab) volume $V$

$$
\frac{d n_{p h}}{d t} \approx 2 \pi \frac{N_{p} N_{e} r_{e}^{2} c}{\gamma^{2} V}
$$

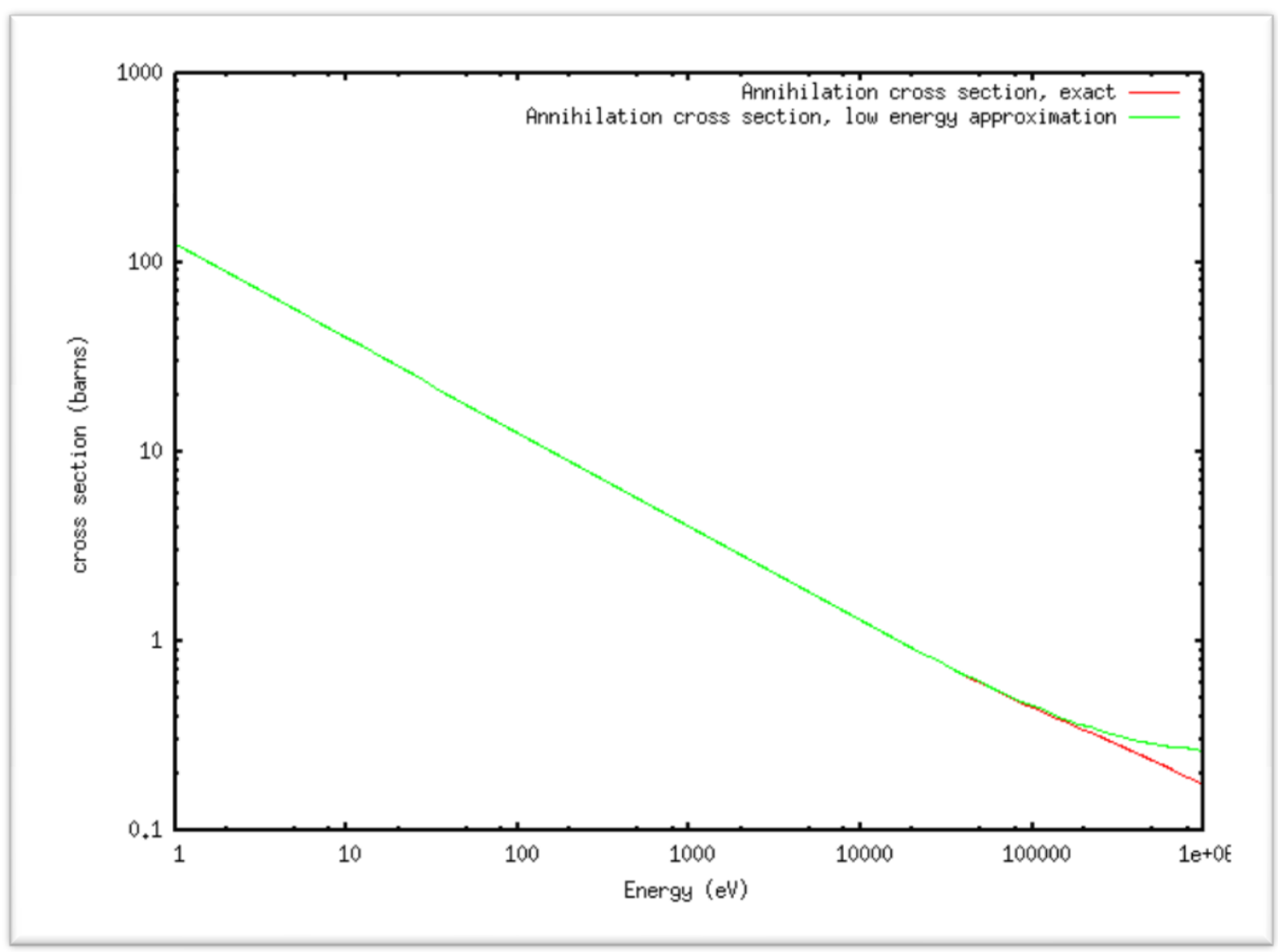

Figure 4: Two photon annihilation cross section for electron-positron interactions with center-of-mass kinetic energy. So long as the kinetic energy is significantly less than the electron rest mass $(511 \mathrm{keV})$, the low energy approximation for the cross section holds and the lifetime will be independent of energy.

Two photon annihilation at rest will produce mono-energetic photons of the electron rest energy $(511 \mathrm{keV})$ in the rest frame of the electron and positron. When boosted to the lab frame, 
the photon energy increases by a factor of $\gamma(1+\beta \cos \theta)$, where $\beta$ is the bunch velocity in the lab relative to the speed of light in vacuum, $\gamma=1 / \sqrt{1-\beta^{2}}$ is the Lorentz factor for the restframe to lab-frame boost, and $\theta$ is the angle of the photon with respect to the direction of beam propagation. When the desired photon energy is significantly higher than the electron rest mass, $\beta$ will be close to 1 and the photon energy in the forward direction is nearly $2 \gamma m_{e} c^{2}$. To achieve the $10 \mathrm{MeV}$ photons for photofission in the forward direction, a $\gamma$ of very close to 10 is needed. For nuclear resonance fluorescence near $2 \mathrm{MeV}$, a $\gamma$ of approximately 2 is required. For precise calculations, the required speed for forward-directed photons of energy $E$ is

$$
\begin{gathered}
\beta=\frac{\left(E /\left(m_{e} c^{2}\right)\right)^{2}-1}{\left(E /\left(m_{e} c^{2}\right)\right)^{2}+1} \\
\gamma=\frac{\left(E /\left(m_{e} c^{2}\right)\right)^{2}+1}{2 E /\left(m_{e} c^{2}\right)}=\frac{E /\left(m_{e} c^{2}\right)}{2}+\frac{1}{2 E /\left(m_{e} c^{2}\right)} .
\end{gathered}
$$

In practice, there will be a finite line width to the boosted annihilation photons. Since annihilation is not occurring at rest but rather at a finite temperature (i.e. momentum spread), the photon energy in the center-of-mass rest frame of the annihilating particles will be increased by the kinetic energy the individual particles in the center-of-mass frame, which will be on the order of the temperature (when expressed in units of energy). In addition, the center-of-mass frame of the annihilating pairs will have a non-zero velocity with respect to the bunch, leading to a spread of relativistic Doppler shifts. Finally, the finite angular acceptance gives a spread of energies to the beam, since photons emitted in different directions will have different Doppler shifts from the boost. In numerical simulations of these effects, the Doppler broadening dominates, such that the energy spread is given by a Gaussian distribution with a standard deviation of $\Delta E=E \sqrt{k_{b} T /\left(2 m_{e} c^{2}\right)}$, although at low temperatures the finite angular acceptance can be noticeable. Beam line widths for co-moving bunches are shown in Figure 5Figure. For head on relativistic collisions where the center-of-mass is in the lab frame, there is no contribution from angular acceptance and only Doppler broadening has a significant contribution to the beam line width. 
PNNL- SA-70285

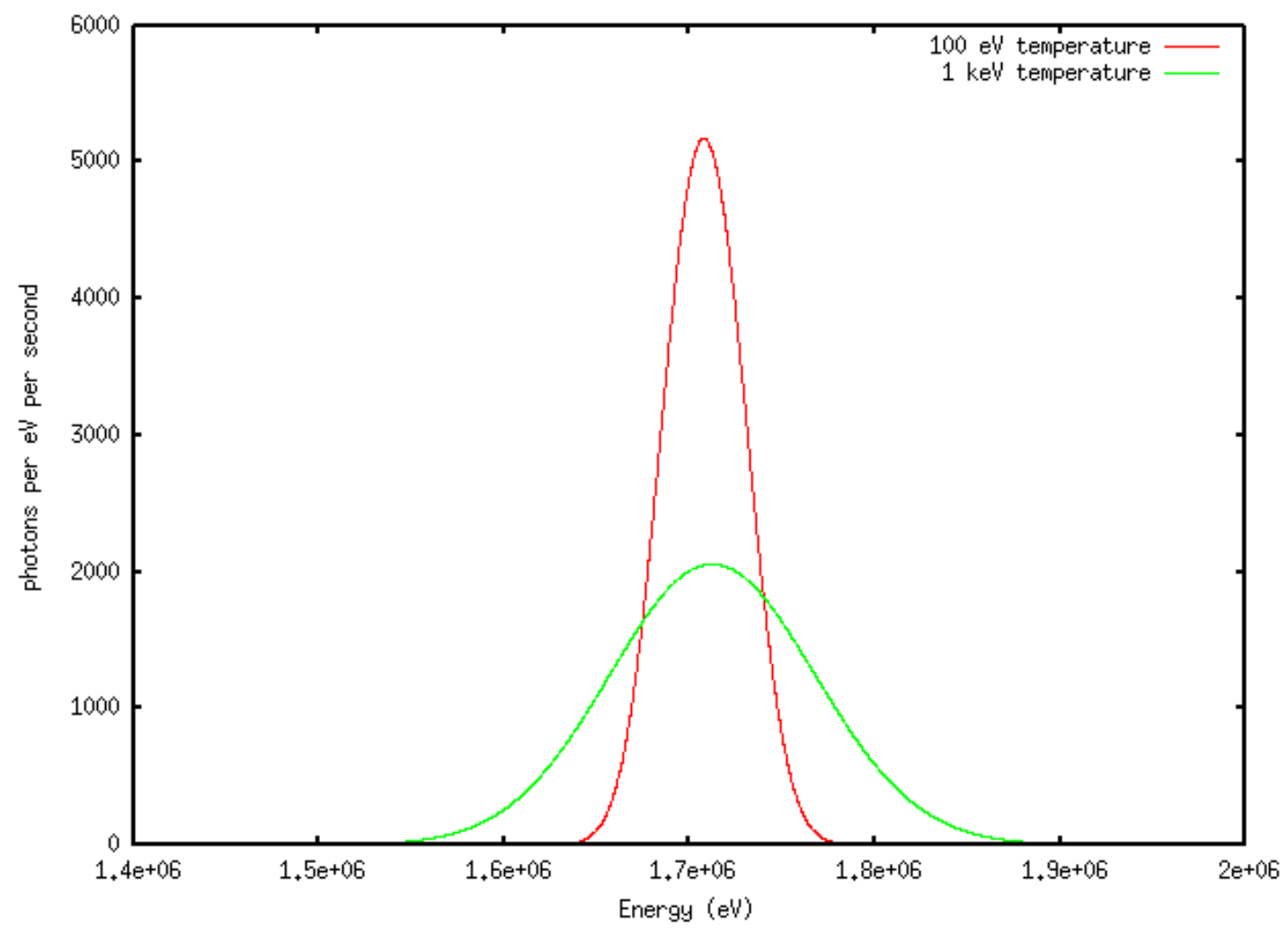

Figure 5: Photon beam energy spread for a $1.73 \mathrm{MeV}$ photon beam from co-propagating annihilation at electron/positron beam temperatures of $100 \mathrm{eV}$ and $1 \mathrm{keV}$. This figure shows the absolute intensity for $10^{10}$ annihilations per second and an angular acceptance of 0.1 radians.

\subsubsection{Bremsstrahlung}

The collision of charged particles gives rise to bremsstrahlung radiation. This is most easily analyzed in the rest frame of the electron-positron bunch, where the system can be treated as an electron-positron plasma. Like-charged collisions have no net electric dipole, the lowest order radiation they can emit is quadrupole. Thus, electron-electron and positron-positron bremsstrahlung is suppressed compared electron-positron bremsstrahlung, and to first order we only need consider opposite-charged collisions at non-relativistic temperatures.

For a non-relativistic beam of positrons moving through a cloud of electrons, we can express the bremsstrahlung power in a simple analytical form

$$
\frac{d P}{d V d \omega}=\left\{\begin{array}{cl}
\frac{64}{3 \sqrt{3}} \rho_{p} \rho_{e}\left(\frac{c}{v_{\mathrm{rel}}}\right) m_{e} c^{2} r_{e}^{3} G\left(v_{\mathrm{rel}}\right) & \omega \leq m_{e} v_{\mathrm{rel}}^{2} \\
0 & \omega>m_{e} v_{\mathrm{rel}}^{2}
\end{array}\right.
$$


where $m_{e}$ is the electron rest mass and $G\left(v_{\text {rel }}\right)$ is the Gaunt factor, a dimensionless number close to unity. In the Born approximation the Gaunt factor is

$$
G\left(v_{\mathrm{rel}}\right)=\frac{\sqrt{3}}{\pi} \ln \left(\frac{m_{e} v_{\mathrm{rel}}^{2}}{4 \hbar \omega}\right)
$$

[Hutchinson 2002]. Note that this differs from the usual electron-ion bremsstrahlung expression because an electron-positron collision behaves dynamically as a single particle collision with a reduced mass $m=m_{e} / 2$.

The differential cross section for excitation of a bremsstrahlung photon of energy $\hbar \omega$ is thus

$$
\frac{d \sigma}{d \omega}=\frac{64}{3 \sqrt{3}}\left(\frac{c}{v_{\text {rel }}^{2}}\right)\left(\frac{m_{e} c^{2}}{\hbar \omega}\right) r_{e}^{3} G\left(v_{\text {rel }}\right)
$$

The total cross section diverges at low energies - the number of low energy photons produced in a collision approaches infinity as the photon frequency approaches zero.

In an electron-positron plasma in thermal equilibrium at temperature $T$, the bremsstrahlung spectral power radiated per unit volume is

$$
\frac{d P}{d V d \omega}=\frac{32}{3} \sqrt{\frac{\pi}{3}} \rho_{p} \rho_{e}\left(\frac{m_{e} c^{2}}{k_{b} T}\right)^{1 / 2} m_{e} c^{2} r_{e}^{3} \exp \left(\frac{-\hbar \omega}{k_{b} T}\right) \bar{g}(\omega, T)
$$

where $k_{b}$ is the Boltzmann constant. The term $\bar{g}(v, T)$ is the Maxwell-averaged Gaunt factor, which in the Born approximation becomes [5]

$$
\bar{g}(\omega, T)=\frac{\sqrt{3}}{\pi} K_{0}\left(\frac{\hbar \omega}{2 k_{b} T}\right) \exp \left(\frac{\hbar \omega}{2 k_{b} T}\right)
$$

where $K_{0}$ is the modified Hankel function. 
PNNL- SA-70285

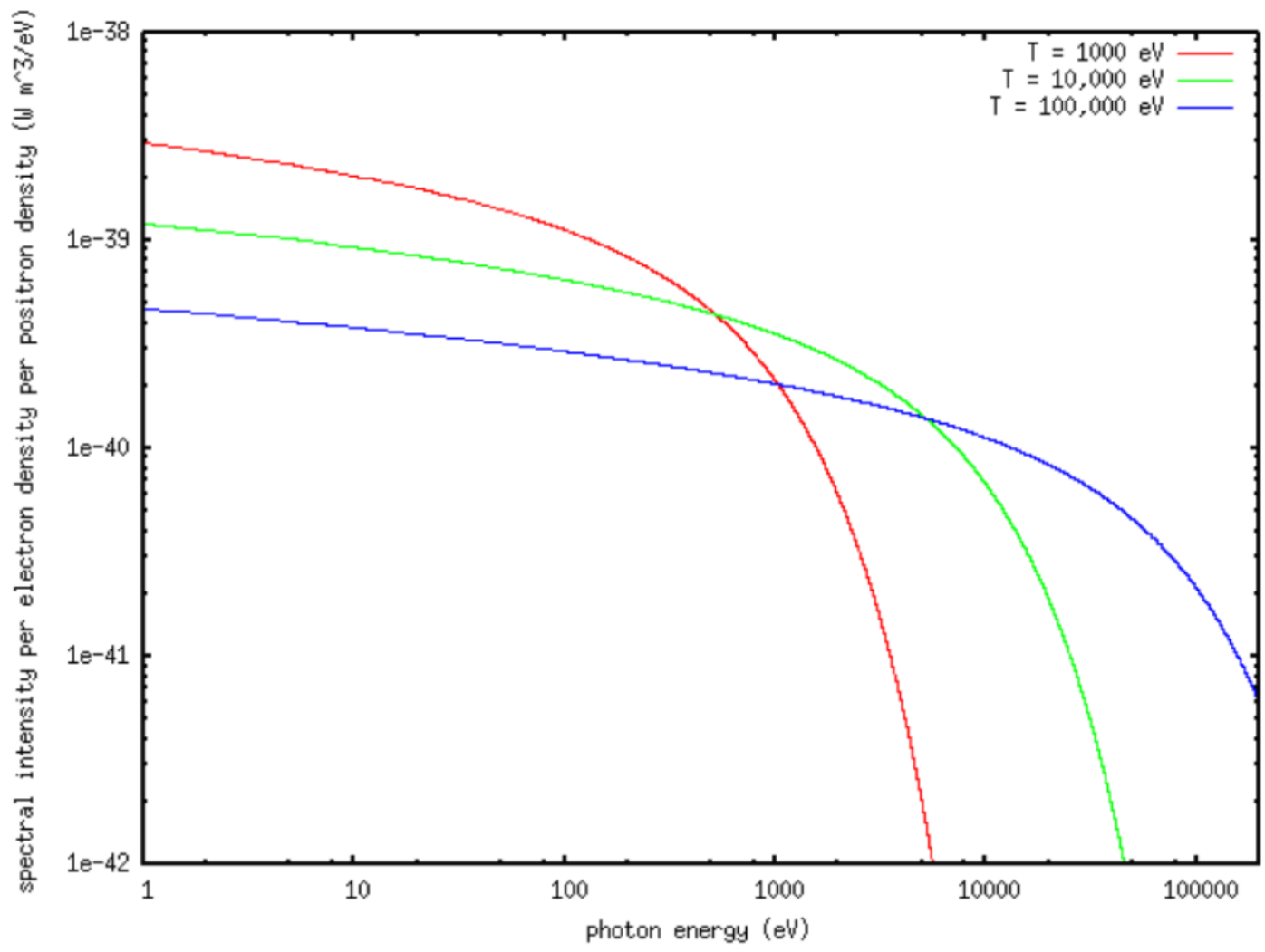

Figure 6: Bremsstrahlung spectral intensity at various beam temperatures.

The total bremsstrahlung power radiated per unit volume can be seen to be proportional to $\sqrt{T}$. The actual integral over frequency is not simple, but evaluates to

$$
\frac{d P_{b r}}{d V}=0.000123 \rho_{p} \rho_{e} m_{e} c^{3} r_{e}^{2} \sqrt{T(\mathrm{eV})} .
$$

Compared to the annihilation power per unit volume, $d P_{a n} / d V=2 \pi \rho_{p} \rho_{e} m_{e} c^{3} r_{e}^{2}$, the ratio of bremsstrahlung power to annihilation power becomes

$$
\frac{P_{b r}}{P_{a n}}=1.95 \times 10^{-5} \sqrt{T(\mathrm{eV})}
$$

which is seen to be small compared to the annihilation power for all non-relativistic temperatures $\left(T \ll m_{e}=511 \mathrm{keV}\right)$. Since both annihilation and bremsstrahlung emissions are isotropic, when boosted to the lab frame the power into a given solid angle is changed by the same amount, and the ratio of the powers remains unchanged.

The bremsstrahlung spectral power falls off rapidly at photon energies significantly greater than the temperature. For a $10 \mathrm{keV}$ plasma boosted such that forward-directed annihilation 
PNNL- SA-70285

gamma rays are blue-shifted to $10 \mathrm{MeV}$, the blue-shifted bremsstrahlung radiation will become negligible at energies significantly greater than about $200 \mathrm{keV}$.

\subsubsection{Positronium formation}

The electron-positron system has a bound state, where the electron and positron orbit in their mutual Coulomb field. This bound system, called positronium, is one of a class of hydrogen-like "atoms" with a binding energy in the ground state of $6.8 \mathrm{eV}$. In its ground state, the electron and positron can have opposite spins to be in a spin zero configuration - the singlet state or para-positronium - or the two particles can have aligned spins for a net spin of one the triplet state or ortho-positronium. Para-positronium decays into two photons with a lifetime of $1.25 \times 10^{-10} \mathrm{~s}$. Two photon decays of ortho-positronium would not conserve parity, the most probable decay mode available to positronium in this triplet state is into three photons with a $1.42 \times 10^{-7} \mathrm{~s}$ lifetime [Vallery, Zitzewitz \& Gidley 2003].

In addition to positronium in the ground state, there are many excited states of positronium, corresponding to the excited states of hydrogen-like atoms. These excited states will be important compared to the ground state when the bunch temperature is much higher than the binding energy. The annihilation lifetime of positronium at angular momentum $l=0$ ( $s$ state) is inversely proportional to the square of the wave function at $r=0$.

$$
\left|\psi_{n, l=0}(0)\right|^{2}=\left(\frac{1}{a_{0}}\right) \frac{1}{2 n^{3}}
$$

from which it can be seen that the annihilation lifetime increases as the cube of the excitation number $n$. For $l \neq 0$, the electron and positron do not overlap and annihilation is negligible. Excited states can also decay via spontaneous emission into states of lower energy. The $2 p \rightarrow 1 s$ transition occurs with a $3.2 \times 10^{-9} \mathrm{~s}$ lifetime.

It should be noted that the individual electron and positron making up an atom of positronium are still subject to annihilation by free electrons and positrons, and by the electrons and positrons in other positronium atoms that may collide with the positronium atom under consideration.

For the purpose of an annihilation photon source, two-photon annihilation of singlet positronium is as good as two-photon annihilation of free electrons and positrons. The resulting photon energy will be shifted by a maximum of $3.4 \mathrm{eV}$ from the electron rest mass (511000 eV) in the rest frame of the bunch. For realistic temperatures, this will be within the line width of the two-photon annihilation line. Three photon annihilation of triplet positronium will produce background radiation outside the main two-photon annihilation line.

In an accelerator system, the mean distance a positronium atom will travel before decay is $\beta \gamma \tau$, where $\tau$ is the lifetime, $\beta$ is the speed relative to the speed of light, and $\gamma$ is the Lorentz $\gamma$ factor defined earlier. Photofission measurements will use $\gamma \approx 10$, while nuclear fluorescence will have $\gamma \approx 2$. If the positronium atom does not undergo decay within the distance the drift distance in which annihilation is occurring, the atom of positronium will pass through this distance intact and encounter the magnetic field that splits the positrons from the electrons. If 
the positronium atom is not ionized by this field, it will pass through and exit the accelerator. In this event, the electron and positron will produce bremsstrahlung, and the positron will annihilate, producing low energy gamma rays.

From this, we can see that para-positronium will likely decay in flight. The mean distance traveled will be $37 \mathrm{~cm}$ at $\gamma=10$ or $6.5 \mathrm{~cm}$ at $\gamma=2$. Only those singlet positronium atoms produced near the very end of the annihilation leg of the beam path will have time to pass through the splitting magnets and enter the material structure of the accelerator. Orthopositronium, however, will have travel a mean distance of about 420 meters at $\gamma=10$ and 74 meters at $\gamma=2$. Consequently, any triplet positronium produced is unlikely to decay within the accelerator and will generate additional background radiation. Positronium in an excited state is also likely to reach the end of the annihilation region where it may pass through the splitting magnetic field.

In thermal equilibrium, the density of positronium atoms in an excited state of principle quantum number $n$ is

$$
\rho_{n, p o s}=\left(\frac{2 \pi \hbar^{2}}{m_{e} k_{b} T}\right)^{3 / 2} n^{2} \rho_{p} \rho_{e} \exp \left(\frac{R_{y}}{2 n^{2} k_{b} T}\right)
$$

where $R_{y}=13.6 \mathrm{eV}$ is the Rydberg constant. In the limit of high temperatures the exponential approaches unity and

$$
\rho_{n, p o s}=\left(\frac{2 \pi \hbar^{2}}{m_{e} k_{b} T}\right)^{3 / 2} n^{2} \rho_{p} \rho_{e}, \quad T \gg R_{y} .
$$

Formally, this diverges such that we end up with an infinite amount of positronium when summing over all excited states. However, there are many reasons why this will not physically be the case. The average radius of a positronium atom in excited state with principle quantum number $n$ and angular momentum quantum number $l$ is $\langle r\rangle=a_{0}\left[3 n^{2}-l(l+1)\right]$. When this radius is larger than the beam radius, we do not expect that quantum state to be in thermal equilibrium. For micron-width beams, this limits us to $n$ on the order of 100 or so.

Further, if the mean time between collisions is significantly less than the lifetime of a state, that state will not be in thermal equilibrium, as it is depopulated faster than it can be filled. If the radiative decay lifetime to that state is shorter than the collisional lifetime, then the states which radiatively feed that state also will not be in thermal equilibrium. If the time spent in the annihilation leg of the accelerator is less than the mean time between collisions, there will be insufficient time to form an equilibrium population of positronium after mixing the electrons and positrons until they are separated by the splitting magnet.

In addition, the electron-positron plasma is not in radiative equilibrium. Since it is optically thin, decay radiation as excited states fall into states of lower energy mostly escape and do not participate in pumping other atoms of positronium up into higher excited states. As a consequence, the transition between states will tend to go from higher to lower levels of 
excitation and the higher excited states will tend to be depopulated compared to lower excited levels.

Finally, there is a practical issue that any states of positronium that are sufficiently loosely bound that they are ionized by the splitting magnetic field do not concern us, as they will not produce unwanted background radiation. From dimensional analysis, we would expect prompt ionization when the positronium encounters an electric field of magnitude $\varepsilon>E /(e\langle r\rangle)$ where $E$ is the binding energy of the state of the positronium. Using $E_{n}=R_{y} /\left(2 n^{2}\right)$ and $\left\langle r_{n, l}\right\rangle=$ $a_{0}\left[3 n^{2}-l(l+1)\right] \approx 3 a_{0} n^{2}$ we find the field needed for ionization of positronium in a state of principle quantum number $n, \varepsilon_{n}>\varepsilon_{a} /\left(12 n^{4}\right)$, where $\varepsilon_{a}=2 R_{y} /\left(e a_{0}\right)=1.71 \times 10^{7}$ statvolt $/ \mathrm{cm}$ is the unit of electric field in the atomic system of units. The electric field arises primarily from the Lorentz transform of the magnetic field in the lab frame used to split the electron-positron plasma into separate electron and positron beams. For a magnetic field of magnitude $B$, the electric field experienced in the rest frame of the positronium atom is $\varepsilon=\gamma \beta B$ (in Gaussian units). Thus, $B_{n}>\varepsilon_{a} /\left(12 \gamma \beta n^{4}\right)$. For electrons of $\gamma=10$, the magnetic field needed to ionize the ground state is $B_{1}>1.43 \times 10^{5}$ gauss; for the first excited states $B_{2}>8.93 \times 10^{3}$ gauss; for the $n=3$ excited states $B_{3}>1.77 \times 10^{3}$ gauss; and for the $n=10$ excited states $B_{10}>14.3$ gauss. At $\gamma=2$, these values increase by a factor of 5.8. It should also be noted that these numbers are mildly conservative, and fields of about $1 / 3$ this strength have been found to cause prompt ionization while rapid ionization through tunneling can still occur at fields of around 1/10 the values given above [Durand and Paidarová 2003].

Thus, the equilibrium concentration of positronium is an upper limit to the amount we expect in the accelerator. For $10 \mathrm{keV}$ temperatures, we find at equilibrium $\rho_{n, p o s}=(3.32 \times$ $\left.10^{-34} \mathrm{~m}^{3}\right) n^{2} \rho_{p} \rho_{e}$, and $\rho_{\text {pos }}=\sum_{n} \rho_{n, \text { pos }} \approx\left(10^{-34} \mathrm{~m}^{3}\right) n_{\text {max }}^{3} \rho_{p} \rho_{e}$. For $n$ limited to no more than 10 or so, we find that the total positronium concentration is bounded above by a number on the order of $\rho_{\text {pos }} \approx 10^{-31} \rho_{p} \rho_{e}$. The number of positronium atoms in a bunch passing through the splitting magnet therefore becomes a number less than $n_{\text {pos }}<\left(10^{-31} \mathrm{~m}^{3}\right) N_{p} N_{e} /\left(\gamma^{2} V\right)$. This can be compared to the number of annihilation photons created per bunch after passing through an annihilation leg of length $L$,

$n_{p h} \approx 2 \pi N_{p} N_{e} r_{e}^{2} L /\left(\gamma^{2} V\right)=\left(5.00 \times 10^{-29} \mathrm{~m}^{2}\right) N_{p} N_{e} L /\left(\gamma^{2} V\right)$. The power of background radiation caused by positronium formation is smaller than $\gamma m_{e} c^{2} n_{\text {pos }}$, because some of the electron and positron kinetic energy is lost as ionization, while the power of annihilation radiation is $\gamma m_{e} c^{2} n_{p h}$. Taking $L$ on the order of 10 meters, we find that the ratio of background power due to positronium formation to the annihilation radiation power is bounded above by

$$
\frac{P_{p o s}}{P_{p h}}<2 \times 10^{-4}
$$

This estimate will clearly change for different temperatures, splitting field strengths, and accelerator lengths, but indicates that the accelerator can be tuned to suppress the positronium contribution to emitted radiation by several orders of magnitude below that of annihilation. For colliding beam annihilation, positronium will not form and can thus be ignored altogether. 
PNNL- SA-70285

\subsection{Research Issues}

\subsection{Accelerator Configuration}

There are three possible configurations for an e+e- collider and all three will be investigated to determine the best solution for both the low energy (NRF for SNM) and high energy (photofission, gamma resonance fluorescence and NRF on explosives) regimes.

\subsubsection{Fixed target}

In this configuration positrons are accelerated and impinge on a fixed target. This is similar to the configuration of a standard bremsstrahlung photon source in some respects, but quite different in two important details. First and foremost, the incident particles are positrons rather than electrons so annihilation can take place between the beam positrons and the electrons in the target material. Second, for this application a low-Z target would be employed to minimize bremsstrahlung and pair production from interactions of the produced photon beam with the target material. Note that these processes are proportional to $Z^{2}$, and thus the rates for these undesirable processes (bremsstrahlung and pair production) drop by a factor of $\sim 600$ when using hydrogen target rather than a tungsten target. Solid (e.g. Be, $\mathrm{LiH}$ ) and gaseous (e.g. H) targets may be considered.

This configuration may be particularly interesting for lower energies (required for NRF measurements of SNM) where the velocity of the positron beam is low and hence the cross section for annihilation is relatively high, while the resulting photons have small pair production cross section and hence secondary bremsstrahlung photon production is suppressed. In addition the beam dynamics for the positron beam are most challenging at low energies and this configuration as one can use a thick target in a single pass configuration, i.e. positrons are produced, accelerated and delivered to the target with no recirculation of the positron beam.

\subsubsection{Colliding beams}

This configuration is the classical high energy physics configuration with electrons and positrons colliding head on. We will discuss here only the symmetric energy case; although asymmetric energies are possible the added complications are unlikely to be worthwhile. When the beam particles annihilate the photons produced are precisely at the energies of the incoming particles. In addition the photons are produced strongly peaked in the beam direction (both forward and backward). An interesting feature of this configuration would be the production of two interrogating beams from a single device, capable of scanning two parallel lanes of traffic or streams of cargo. The photon beams produced would have the narrowest energy spread achievable, the only spreading being due to Doppler broadening from the energy spread of the incoming particle beams. This configuration suffers from lower cross section for annihilation which scales as the inverse of the center-of-mass energy. However, this configuration allows for a significant enhancement of the accelerator luminosity through the use of a low beta insertion at the collision point (this amounts to a strong focusing of the beams at the collision point). There may be bremsstrahlung or beamsstrahlung (this is a similar process 
where the field of the opposing beam accelerates the particles), but its contribution is not known at this time.

\subsubsection{Co-moving beams}

This is the original configuration we considered where the two beams would have equal energies and move in the same direction. The annihilation photons are then boosted due to the velocity of the center of mass. This produces a strongly forward focused beam peaked towards the center of mass energy. However, there is considerable Doppler broadening, particularly at lower energies where the ratio of the beam energy to the electron rest mass is not large. The cross section is maximal for annihilation in this configuration and the total photon production will increase as the "collision" region is lengthened. However, controlling the extremely low energy beams required to produce photons in the $\sim 2 \mathrm{MeV}$ range required for NRF of SNM is difficult. The net effect is likely to be larger than desired energy spread as well as poor focusing, and hence lower luminosity. There are a number of research issues related to managing the two beams and bringing these into collision. Initial conceptual discussions have identified solutions to these issues.

\subsection{Positron source}

As mentioned previously, the production of positrons will limit the available photon flux. There are several options for producing positrons, each with its pros and cons. Ultimately we expect that the total positron beam intensity produced will be the deciding metric to choose between options. It is important to note that positron production rate alone is not a suitable metric as capture and acceleration efficiencies can vary by several orders of magnitude.

\subsubsection{Radioactive sources}

The most common positron source is ${ }^{22} \mathrm{Na}$. Sources can be obtained commercially with activities of $100 \mathrm{mCi}$. The end point energy of the decay is $2.2 \mathrm{MeV}$. In order to capture the positrons it is typical that the positrons are moderated using Tungsten foils. This process has an efficiency of $10^{-3}-10^{-2}$, but the resulting positron beam will have a low emittance and momentum spread. The net result is a rate of about $10^{7}$ positrons per second per $\mathrm{Ci}$. A 100 $\mathrm{mCi}$ class source is about $\$ 100 \mathrm{k}$. With a half-life of 2.6 years, the source would need to be replaced or augmented about once a year.

Other long-lived positron emitters could also be considered, but generally have undesirable gamma ray emissions that would require additional shielding.

\subsubsection{Electroproduction}

This process involves using 10-20 MeV electrons impinging on a solid target, resulting in pair production. Using $20 \mathrm{MeV}$ electrons results in higher positron production rates, but there is also sufficient energy to produce undesirable neutron backgrounds. These neutrons can further lead to activation of materials in the target area. Using $10 \mathrm{MeV}$ electrons reduces the neutron production to a negligible level at the expense of positron production rate. After moderation and 
capture the efficiency of electroproduction of positrons is about $10^{-7}$ positrons per incident electron. Positron production rates of $10^{8}$ per second could be achieved with a $\mathrm{mA}$ electron beam.

\subsubsection{Proton beam produced short-lived positron sources}

This method would produce a very high activity positron emitting source in situ using a proton beam. Proton accelerators with energies up to $9 \mathrm{MeV}$ with beam currents in the range of a $\mathrm{mA}$ are commercially available in portable configurations for production of short-lived medical isotopes. There are a number of proton reactions that could be employed. Here we describe the most promising reaction involving protons impinging on a ${ }^{19} \mathrm{~F}$ target. The resulting isotope, ${ }^{20} \mathrm{Ne}$, decays via alpha emission to ${ }^{16} \mathrm{O}$. The excited states produced are forbidden to decay to the ground state by gamma emission (they are all $0^{+}$states), and hence the de-excitation occurs via internal conversion with emission of an e+e-pair with a lifetime of the $70 \mathrm{fs}$. The resulting decay product is stable. The result is a source which will be "switched off" nearly instantaneously when the proton beam is turned off. The initial reaction requires a proton beam with an energy of only $2 \mathrm{MeV}$ while the resulting e+e- pair share $6.05 \mathrm{MeV}$ of energy from the de-excitation. The resulting positron spectrum is peaked around $2.5 \mathrm{MeV}$, ranging up to $5 \mathrm{MeV}$. After moderation, the expected slow positron rate is $10^{7}-10^{8}$ per second for a $1 \mathrm{~mA}$ proton beam. This process has been published and patented by BTG, Inc. [Guardala, Farrell, \& Dudnikov 2001].

\subsection{Accelerator lattice design}

Once a configuration is selected for the accelerator (head-on collider, fixed target, comoving beams) a suitable accelerator lattice must be designed. Although there is the possibility that a single-pass, fixed target configuration may be feasible, it is expected that a recirculating accelerator will be required to store the positrons, and quite probably the electrons. In order to maximize positron capture efficiency it will be critical to have a large acceptance accelerator lattice design. The qualities required to maximize acceptance will be low dispersion and small beta functions. coupled with a large physical aperture. This is quite different from the situation with electron linacs where the beam is small both in transverse dimensions and momentum spread.

Another consideration for a variable energy machine is the transition energy. In simple terms, accelerators maintain stability through the interplay of orbit length in the machine and RF accelerating field. Slower particles are bent more strongly in the arcs and travel a shorter path around the ring, arriving in the accelerating cavity earlier than the faster moving particles. The $\mathrm{RF}$ phase is adjusted so that the slower moving particles are accelerated while the faster moving particles are decelerated. At the transition energy of an accelerator the orbit length is identical for all velocities and hence there beam becomes unstable. Complex methods have been developed to "jump" transition, but these are difficult and frequently lead to beam losses. Therefore it is essential to select a transition energy that is either above or below the energy range of interest for the accelerator. An alternative solution is a design that results in an imaginary transition energy ("imaginary" in the mathematical sense that the transition energy for an accelerator is a complex number and in these designs has no real component). 
The basic layout envisioned consists of two 180 degree arc sections with two straight sections connecting them. One straight section will be utilized for injection of positrons, perhaps via electroproduction from a target inserted into the edge of the electron beam. The other straight section will be used for collisions. The requirements for the optics in these sections will differ depending on the functionality required. For example, if electroproduction on a foil target is planned, one would want a "low beta" insertion at the target where the beams are highly focused at the target location, but diverge quickly to either side of the target. This would also be the case for the collision point in a head-on colliding beam configuration. In contrast, for the comoving beam configuration one wants a modestly low beta over several meters of straight section so that the beams are as dense as can be achieved while that travel together along the interaction section. Unlike the typical triplet quadrupole configuration of a low beta insertion, maintaining focusing over several meters of length will require the use of a solenoid or helical quadrupole magnet.

\subsubsection{Acceleration}

The final element in the accelerator design will be the accelerating cavities used. The cavity aperture will need to be large to provide high capture efficiency for positrons. This suggests superconducting RF (SRF) cavities rather than conventional copper cavities or RF quadruples that have small apertures. There are large aperture cavity designs that do not require cryogenic cooling which will also be investigated for this application. Basic accelerator design must proceed first in order to determine the desired RF frequency range, which in turn will help define suitable technology.

\subsection{Conclusions}

Production of photon beams from positron annihilation in flight is being investigated as an alternative to bremsstrahlung and laser Compton backscatter for interrogation of cargo for SNM. There are two regimes of interest, each with its own requirements and challenges. The first is photofission energies ( $6 \mathrm{MeV}$ up to $20 \mathrm{MeV}$ ) where beam energy spread is not critical, but suppression of photons below $\sim 6 \mathrm{MeV}$ could reduce dose rates to cargo considerably, enabling this technique to be deployed more readily under existing federal regulations. It is worth noting that this region is also interesting for explosives detection using gamma resonance absorption and nuclear resonance fluorescence techniques, in which case beam energy spread becomes much more important as one is searching for very narrow $(\mathrm{eV}$ to $150 \mathrm{eV})$ resonances. The second region of interest lies near $2 \mathrm{MeV}$ where there are NRF signatures for SNM. Here photon beam energy spread is critical to enhance the photon flux within the extremely narrow resonances.

We have outlined the relevant physics processes involved and conclude that the annihilation process has sufficient cross section to be a feasible photon source given achievable positron source strengths.

\subsection{References}


1) J.T. Caldwell, E.J. Dowdy, R.A. Alvarez, B.L. Berman and P. Meyer, "Experimental Determination of Photofission Neutron Multiplicities for ${ }^{235} \mathrm{U},{ }^{236} \mathrm{U},{ }^{238} \mathrm{U}$, and ${ }^{232} \mathrm{Th}$ Using Monoenergetic Photons", Nuc. Sci. and Eng., Vol. 73, pg. 153-163 (1980).

2) P. A. M. Dirac., "The Quantum Theory of the Electron," Proceedings of the Royal Society of London A, Vol. 117, pg. 610-624, 1928; "The Quantum Theory of the Electron, part II", Proceedings of the Royal Society of London A, Vol. 118, pg. 351-361, 1928.

3) Ph. Durand and I. Paidarová, "Ionization of the hydrogen atom: from weak to strong static electric fields," The European Physical Journal D, Vol. 26, pg. 253-259, 2003.

4) N. A. Guardala, J. P. Farrell, and V. Dudnikov, "An intense, compact fourth-generation positron source based on using a $2 \mathrm{MeV}$ proton accelerator," AIP Conference Proceedings, Vol. 576, pg. 741-744 (2001).

5) I. H. Hutchinson, Principles of Plasma Diagnostics, Second Edition, Cambridge University Press, Cambridge, 2002.

6) I. N. Meshkov, "LEPTA project: generation and study of positronium in directed fluxes," Nuclear Instruments and Methods in Physics Research B, Vol. 221, pg. 168-173 (2004).

7) A. Mikhailichenko, "Damping ring for generation of positroniums and investigation of Fermi degeneration in moving beams," Particle Accelerator Conference, 2003. PAC 2003. Proceedings of the 200 Particle Accelerator Conference, vol.3, no., pp. 1828-1830 vol.3, 12-16 May 2003

URL: http://ieeexplore.ieee.org/stamp/stamp.jsp?arnumber=1288684\&isnumber=28731

8) A. Mikhailichenko, "On the physical limitations to the lowest emittance (toward Colliding electron-positron crystalline beams) ," $7^{\text {th }}$ Advanced Accelerator Concepts Workshop, AIP Proceedings 398, p. 294. CLNS 96/1437, Cornell, 1996.

9) A. Ore and J. L. Powell, "Three Photon Annihilation of an Electron-Positron Pair," Physical Review, Vol. 75, pg. 1696 - 1699, 1949.

10) R. S. Vallery, P. W. Zitzewitz, and D. W. Gidley, "Resolution of the OrthopositroniumLifetime Puzzle," Physical. Review. Letters, Vol. 90, article 203402, 2003. 


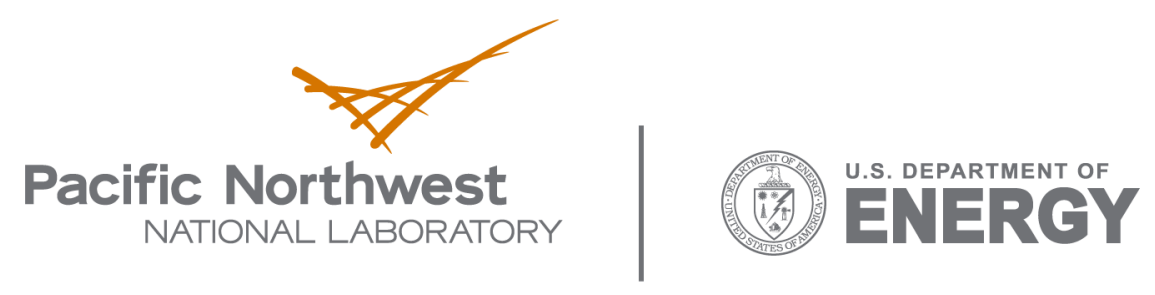

902 Battelle Boulevard

P.O. Box 999

Richland, WA 99352

1-888-375-PNNL (7665)

www.pnl.gov 